\title{
IMPACT OF DOMAIN ANALYSIS ON REUSE METHODS
}

\section{Kathy Gilroy}

Software Productivity Solutions, Inc.

SPS is performing a study for the US Army CECOM on the impact of domain analysis on reuse methods. Domain analysis is the first activity that should be performed in the development of reusable software. It identifies the commonalities between systems within a given problem domain (such as navigation systems or database management). In the software arena these commonalities are implemented as software components that can be reused by new systems within that application domain. The objectives of the study are to develop an approach that makes domain analysis practical and effective for the Army, to reinforce the importance of domain analysis for software reuse programs, and to summarize and coalesce domain analysis information into a single reference source. Existing methods and tools are being analyzed, critical issues identified, and key automation issues addressed. Based on these, a methodology and set of guidelines for domain analysis are being developed. Potential automated tools will be identified for each activity in the methodology. 


\section{Problem Statement}

\section{What is Domain Analysis?}

- The first activity which should be performed in the development of reusable software

- Identifies commonalities between systems within a given problem domain

- Commonalities implemented as software components reused by new systems within that domain

Little Data Available About Domain Analysis

- Few have been done to date

- Importance only recently identified

- Process is difficult and expensive

- Potential payoff not yet known

- "Bad" analysis decreases ability to reuse

- Well-defined methods non-existent

- "Goodness" criteria non-existent

- Recent ad hoc efforts provided little feedback

- No tools support entire analysis process 


\section{Objectives}

- Develop approach that makes domain analysis practical and effective for Army

- Reinforce importance of domain analysis for software reuse program

- Summarize and coalesce domain analysis information into single reference source

\section{Approach}

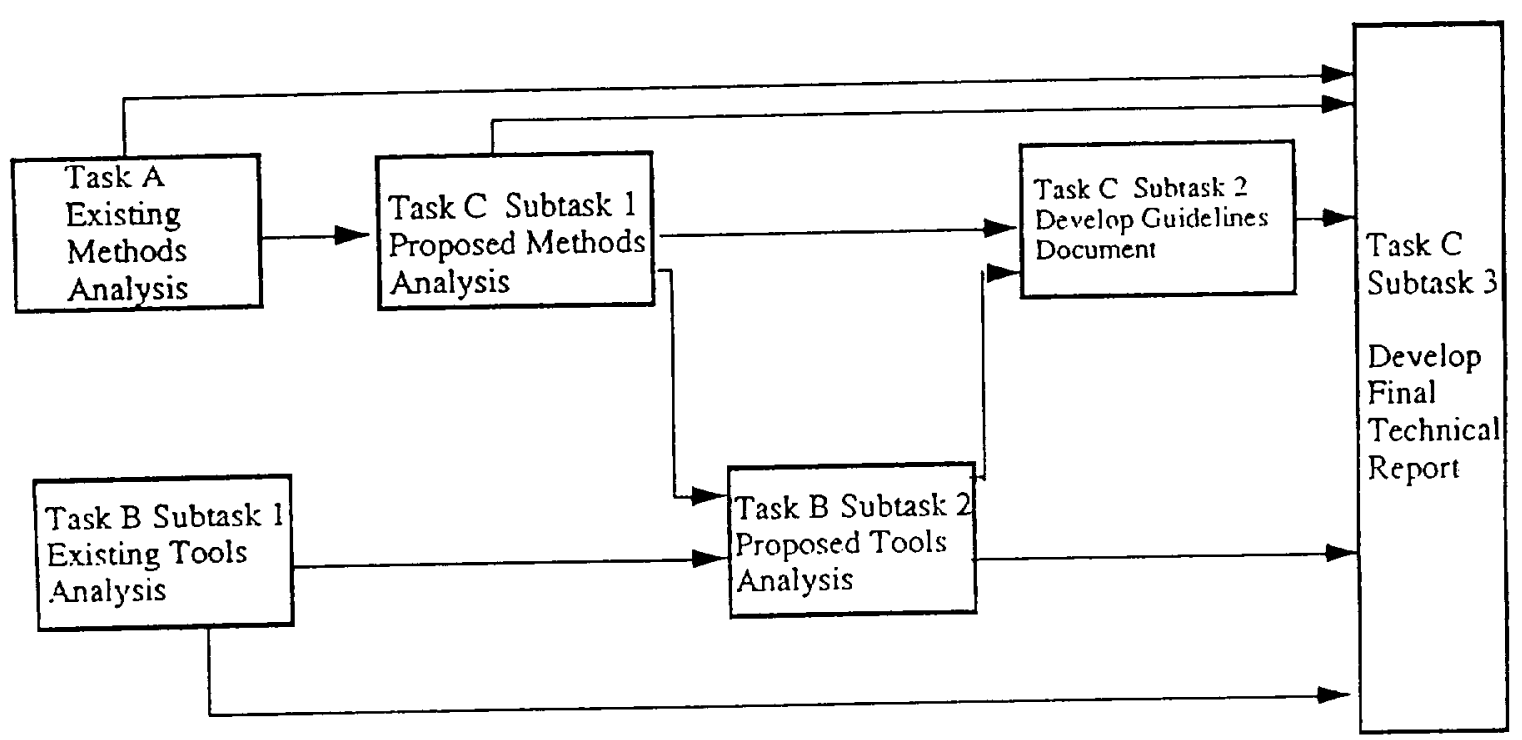

Task Interrelationships 


\section{Approach}

\section{Existing Methods Analysis}

- Identify criteria for evaluating domain analysis approaches

- Survey existing methods for domain analysis and relate to criteria

- Develop description of desirable characteristics of a domain analysis

- Identify critical issues and assess risks involved

\section{Identify Critical Issues}

- Development methods

- Development languages

- Development tools

- Development personnel

- Application systems

- Domain analysis techniques

- Evaluation and validation

- Domain maintenance 


\section{Approach}

\section{Address Key Automation Issues}

- Expert knowledge acquisition and use

- Domain analysis products standardization

- Data organization, storage and retrieval

- Reusable component library interfaces

- Integration with software development environments and reuse tools

- Feasibility of automation and/or tool maturity level

\section{Proposed Methods Development}

- Postulate alternative approaches to domain analysis for DoD Ada applications

- Select one or more approaches for further development

- Provide consistent, cohesive and complete description of proposed method; address the following:

- strategies and paradigms for domain analysis

- process model for domain analysis

- methods for each identified activity

- products of domain analysis activities

- resources required for domain analysis activities 


\section{Approach}

\section{Process Model for Domain Analysis}

- Within context of three distinct but integrated processes:

- development of reusable components

- reuse of components

- development of application software

\section{Proposed Tools Identification}

- For each activity in the proposed methodology:

- identify existing or potential automated tools

- describe how they support the activity

- assess the importance of automating the activity

- assess the feasibility of automation or the maturity level of existing tools

- make recommendations for acquisition or development 


\section{Approach}

\section{Develop a Set of Guidelines}

- Use results of research and analysis of existing methods and tools

- Develop guidelines for conducting domain analysis and applying results during software development; document will contain:

- recommendations for methodology and tools to perform domain analysis

- relationship of domain analysis methodology and tools to overall development methodology and tools

- recommendation for addressing critical issues and risks in using domain analysis

- recommendations for future $R \& D$ in domain analysis 
